\title{
ADVANCES IN CROSS-ENTROPY METHODS
}

\author{
THOMAS TAIMRE
}

(Received 24 June 2009)

2000 Mathematics subject classification: primary 65C05; secondary 68U20, 90C27, 68W20, 62M05. Keywords and phrases: cross-entropy, importance sampling, rare events, optimization.

The cross-entropy method is an established technique for solving difficult estimation, simulation, and optimization problems. The method has its origins in an adaptive importance sampling procedure for rare-event estimation published by Rubinstein in 1997 [1]. In that publication, the adaptive procedure produces a parametric probability density function whose parameters minimize the variance of the associated likelihood ratio estimator. This variance minimization can also be viewed as minimizing a measure of divergence to the minimum-variance importance sampling density over all members of the parametric family in question.

Soon thereafter it was realized that the same adaptive importance sampling procedure could be used to solve combinatorial optimization problems [3] by viewing the set of solutions to the optimization problem as a rare event. This realization led to the debut of the cross-entropy method in 1999 [2], where it was introduced as a modification to the existing adaptive importance sampling procedure, with a different choice of directed divergence measure, in particular, the Kullback-Leibler cross-entropy.

The contributions of this thesis are threefold. Firstly, in a review capacity, it provides an up-to-date consolidation of material on the cross-entropy method and its generalizations, as well as a collation of background material on importance sampling and Monte Carlo methods. The reviews are elucidated with original commentary and examples. Secondly, two new major applications of the cross-entropy methodology to optimization problems are presented, advancing the boundary of knowledge on cross-entropy in the applied arena. Thirdly, two contributions to the methodological front are: (a) an original extension of the generalized cross-entropy framework which enables one to construct state- and time-dependent importance sampling algorithms; and (b) a new algorithm for counting solutions to difficult binary-encoded problems.

Thesis submitted to the University of Queensland, October 2008. Degree approved, May 2009. Supervisor: Professor Dirk P. Kroese.

(C) 2009 Australian Mathematical Publishing Association Inc. 0004-9727/2009 \$16.00 


\section{References}

[1] R. Y. Rubinstein, 'Optimization of computer simulation models with rare events', European J. Oper. Res. 99 (1997), 89-112.

[2] R. Y. Rubinstein, 'The cross-entropy method for combinatorial and continuous optimization', Methodol. Comput. Appl. Probab. 1 (1999), 127-190.

[3] R. Y. Rubinstein and D. P. Kroese, The Cross-Entropy Method: A Unified Approach to Combinatorial Optimization, Monte-Carlo Simulation, and Machine Learning (Springer, New York, 2004).

THOMAS TAIMRE, School of Mathematics and Physics, The University of Queensland, Brisbane 4072, Australia e-mail: ttaimre@maths.uq.edu.au 